\title{
Hydrologic data for the Eastland Woolen Mill Superfund Site, Penobscot County, Corinna, Maine, March through June 1999
}

U.S. GEOLOGICAL SURVEY

Open-File Report 01-212

Prepared in cooperation with the

U.S. Environmental Protection Agency

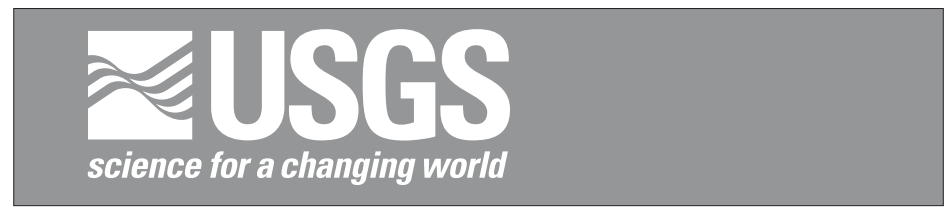




\section{Hydrologic data for the Eastland Woolen Mill Superfund Site, Penobscot County, Corinna, Maine, March through June 1999}

By Martha G. Nielsen, Robert W. Dudley, and Camille S. Parrish

U.S. GEOLOGICAL SURVEY

Open-File Report 01-212

Prepared in cooperation with the

U.S. Environmental Protection Agency 


\section{U.S. DEPARTMENT OF THE INTERIOR GALE A. NORTON, Secretary}

U.S. GEOLOGICAL SURVEY

Charles G. Groat, Director

The use of firm, trade, and brand names in this report is for identification purposes only and does not constitute endorsement by the U.S. Government

For additional information write to:

District Chief

U.S. Geological Survey

26 Ganneston Drive

Augusta, ME 04330

http://me.water.usgs.gov
Copies of this report can be purchased from:

U.S. Geological Survey

Branch of Information Services

Box 25286

Federal Center

Denver, CO 80225 


\section{CONTENTS}

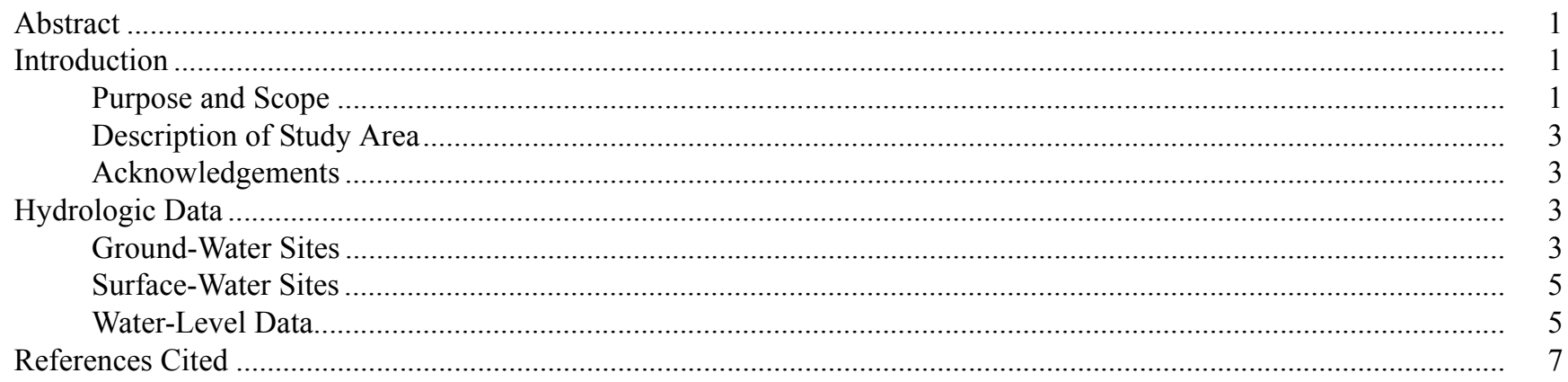

APPENDIX 1. Daily water-level data in wells at the Eastland Woolen Mill Superfund Site, Corinna, Maine, March 19 through June 11, 1999

\section{FIGURES}

1. Map showing location of the Eastland Woolen Mill Superfund Site, Corinna, Maine

\section{TABLES}

1. Description of monitoring wells at the Eastland Woolen Mill Superfund Site, Corinna, Maine

2. Surface-water site information at the Eastland Woolen Mill Superfund Site, Corinna, Maine

3. Discrete water levels measured in wells and at surface-water sites at the Eastland Woolen Mill Superfund Site, Corinna, Maine, from March to June 1999.

\section{CONVERSION FACTORS, AND VERTICAL DATUM}

CONVERSION FACTORS

\begin{tabular}{ccl}
\hline Multiply & By & To Obtain \\
\hline foot (ft) & 0.3048 & meter \\
\hline
\end{tabular}

\section{VERTICAL DATUM}

Sea Level: In this report "sea level" refers to the National Geodetic Vertical Datum of 1929 (NGVD of 1929) - a geodetic datum derived from a general adjustment of the first-order level nets of the United States and Canada, formerly called Sea Level Datum of 1929. 



\section{HYDROLOGIC DATA FOR THE EASTLAND WOOLEN MILL SUPERFUND SITE, PENOBSCOT COUNTY, CORINNA, MAINE, MARCH THROUGH JUNE 1999}

\begin{abstract}
Hydrologic data were collected at the Eastland Woolen Mill Superfund Site, Corinna, Maine, from March 19, 1999 through June 11, 1999 as part of a study to formulate a geologic characterization and conceptual model of this study area. Data-collection consisted of measurements of water-surface elevations at 7 surface-water sites and 20 wells.
\end{abstract}

\section{INTRODUCTION}

The Eastland Woolen Mill Superfund Site has been designated by the U. S. Environmental Protection Agency (USEPA) as a Superfund site under the Comprehensive Environmental Response, Compensation, and Liability Act of 1980 (CERCLA). The Eastland Woolen Mill Superfund Site, referred to in this report as "the site", is located in Penobscot County, Corinna, Maine (fig. 1).

The Eastland Woolen Mill produced fine woolens and blended dyed fabrics from 1912 until 1996. The mill used a variety of dense non-aqueous phase liquids (chlorinated solvents, primarily chlorobenzenes) that were disposed of or stored at the mill site and surrounding locations. These chemicals have migrated through surficial sediments and into fractured bedrock, contaminating ground water (Acheron, 1994; Harding Lawson Associates, 1999). Studies of contamination at the site began in 1983. The USEPA, the U.S. Army Corps of Engineers, and sub-contractor Harding Lawson Associates assessed the extent of the contamination plume in 2000 and have begun remedial action at the site.
The U.S. Geological Survey (USGS) in cooperation with the USEPA began data-collection activities to provide information on the geohydrologic setting at the site as a basis for designing appropriate remedial actions and supporting studies within the study area. A conceptual ground-water model for the site has been constructed (Mack and Dudley, 2001), and geophysical data collected at the site also has been published (Hansen and others, 2001). As part of the overall goal of providing information on the hydrology at the site, the data network described in this report was established to measure water levels in surface-water bodies and ground-water wells.

\section{Purpose and scope}

The purpose of this report is to present hydrologic data collected from March 19, 1999 through June 11, 1999, at the Eastland Woolen Mill Superfund Site in Corinna, Maine. These hydrologic data consist of discrete measurements of water levels at 7 surface-water sites, and both continuous and discrete ground-water-level data in 20 wells. 


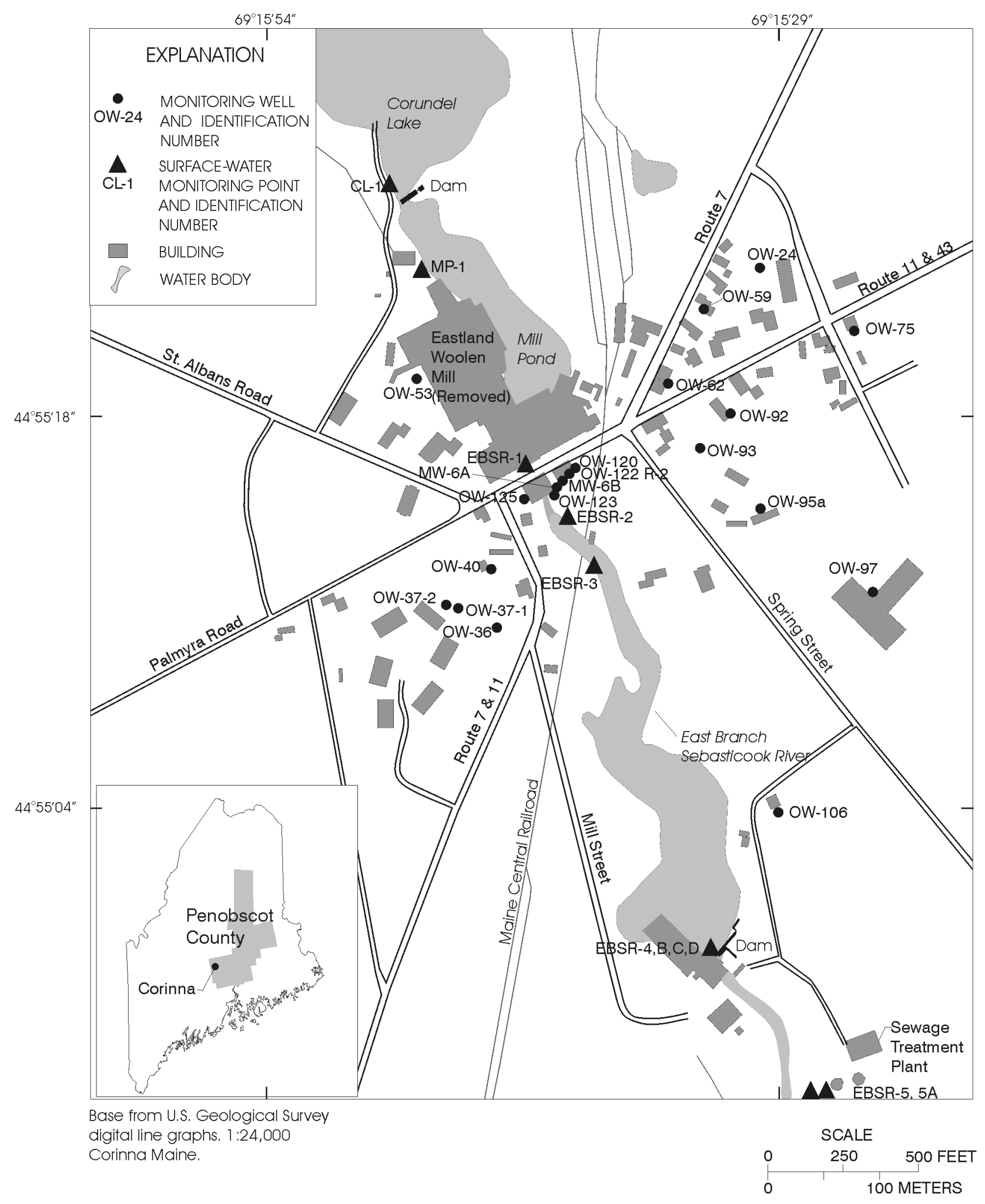

Figure 1. Location of the Eastland Woolen Mill Superfund Site, Corinna, Maine 


\section{Description of study area}

The town of Corinna, in central Maine, is located in a valley formed by the East Branch of the Sebasticook River (fig. 1), which is a tributary to the Kennebec River. The river is dammed in several places to form a number of ponded areas including Corundel Lake and Mill Pond. The town center and most commercial and residential buildings are in the valley lowlands. The former Eastland Woolen Mill, built directly over the river in the center of town (fig. 1), operated from 19121996 and is believed to be the primary source of contamination to the bedrock aquifer adjacent to the river (Harding Lawson Associates, 1999).

There are two aquifers in the study area: a surficial till aquifer and a fractured bedrock aquifer. No significant sand and gravel aquifers have been mapped in the study area. The till aquifer is about $20 \mathrm{ft}$. thick in most places (Harding Lawson Associates, 1999). Throughout most of the study area the water table is in the surficial aquifer. The bedrock aquifer consists of a generally highlyfractured shaley metasediment and siltstone (Osberg and others, 1985; Mack and Dudley, 2001).

\section{Acknowledgments}

The authors thank the individuals who assisted with the data-collection, including Jean Firth, Brian Beneski, and Gordon Fuller with the Maine Department of Environmental Protection (MDEP).

\section{HYDROLOGIC DATA}

The data-collection network consists of ground-water wells and sites for measuring lake and river water-surface elevations.

\section{Ground-water sites}

The ground-water data-collection network consists of 20 observation wells, which include monitoring wells drilled for the study, commercial wells, and residential domestic wells (table 1). Two wells were completed in the surficial till aquifer and the remaining eighteen were drilled into the bedrock aquifer.

The wells are identified with three sets of identifiers: project identifiers, local well numbers, and USGS identification numbers (table 1). The project identifier represents a physical location. For example, observation well 62 (OW-62), is numbered according to the lot number on which the well is located. The project identifiers are used to label the wells on figure 1 . The local well identifiers represent the county and well number. The first part of the identifier contains two letters that denote the state and two letters that denote the county in which the well was drilled, a "W" to identify a well and the number indicates how many wells preceded it. For example, ME-PEW 612 represents Maine, Penobscot County, well number 612. USGS identification numbers for wells are based on the grid system of latitude and longitude. Each number consists of 15-digits. The first six digits denote the degrees, minutes, and seconds of latitude; the next seven digits denote degrees, minutes, and seconds of longitude. The last two digits (assigned sequentially) identify wells or other sites within a 1-second grid. These latitude-longitude identification numbers are the primary identifiers in the USGS ground-water site inventory (GWSI) data base. 


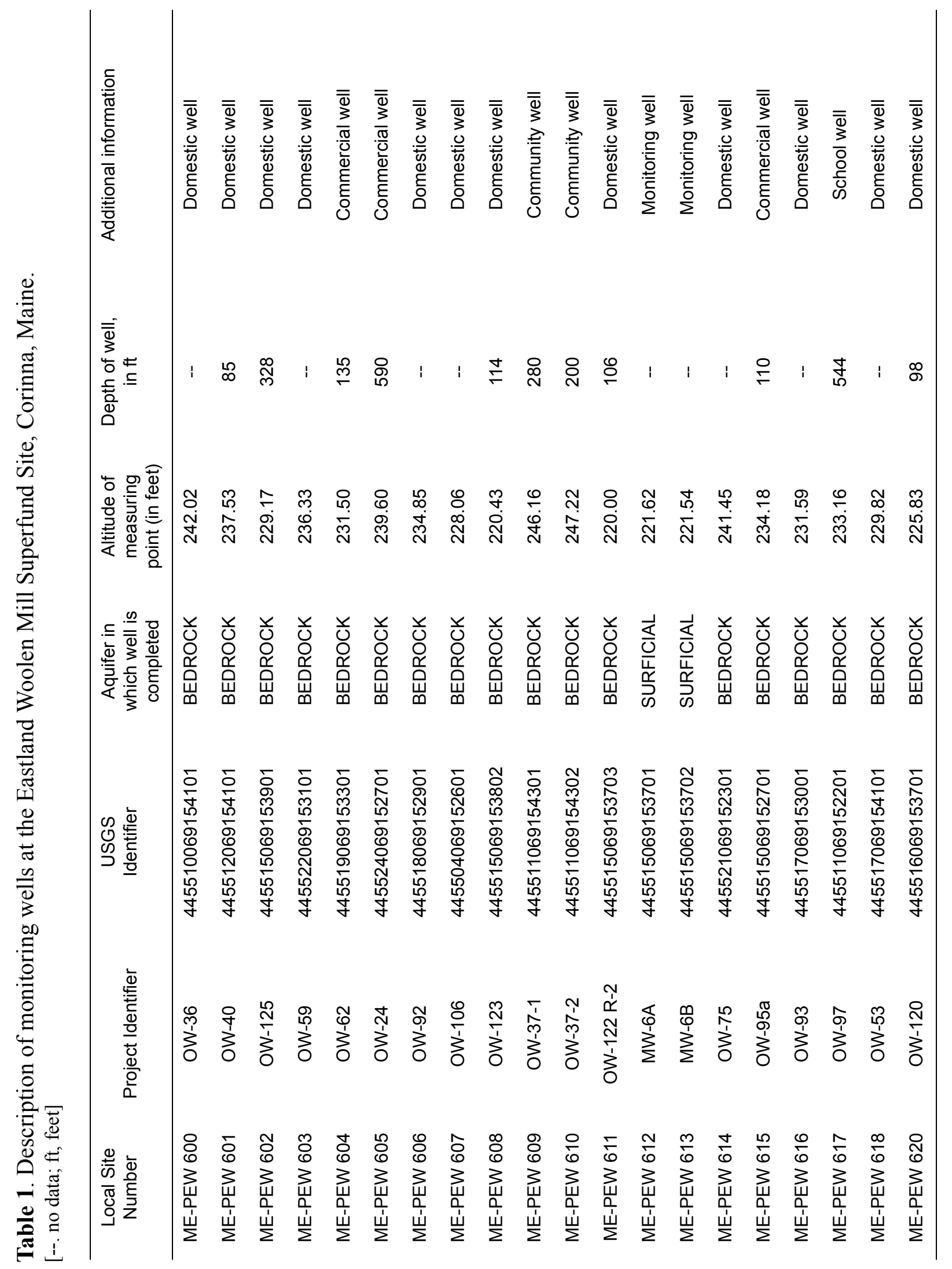

4 Hydrologic Data for the Eastland Woolen Mill Superfund Site, Penobscot County, Corinna, Maine, March through June 1999 
Table 2. Surface-water site information at the Eastland Woolen Mill Superfund Site, Corinna, Maine.

\begin{tabular}{|c|c|c|c|c|}
\hline $\begin{array}{l}\text { Site } \\
\text { Number }\end{array}$ & Station ID & Site Name & $\begin{array}{l}\text { Altitude of } \\
\text { Measuring Point } \\
\text { (in feet) }\end{array}$ & Notes \\
\hline CL-1 & 445527069154701 & Corundel Lake & 231.17 & Located by big rock upstream of dam \\
\hline MP-1 & 445523069154301 & Mill Pond & 230.05 & $\begin{array}{l}\text { Located off edge of first white metal } \\
\text { building }\end{array}$ \\
\hline EBSR-1 & 445516069153901 & $\begin{array}{l}\text { East Branch } \\
\text { Sebasticook River }\end{array}$ & 228.96 & Over bridge downtown (since removed) \\
\hline EBSR-2 & 445515069153801 & $\begin{array}{l}\text { East Branch } \\
\text { Sebasticook River }\end{array}$ & 220.35 & -- \\
\hline EBSR-3 & 445513069153701 & $\begin{array}{l}\text { East Branch } \\
\text { Sebasticook River }\end{array}$ & 222.46 & $\begin{array}{c}\text { Marked on left bank wall of } 2 \text { nd tier of } \\
\text { bridge abutment }\end{array}$ \\
\hline EBSR-4 & 445459069153001 & $\begin{array}{l}\text { East Branch } \\
\text { Sebasticook River }\end{array}$ & 212.14 & $\begin{array}{l}\text { Point moved several times due to } \\
\text { vandalism and beaver activity. }\end{array}$ \\
\hline EBSR-4B & 445459069153001 & $\begin{array}{l}\text { East Branch } \\
\text { Sebasticook River }\end{array}$ & 215.06 & (See comment for EBSR-4) \\
\hline EBSR-4C & 445459069153002 & $\begin{array}{l}\text { East Branch } \\
\text { Sebasticook River }\end{array}$ & 206.65 & (See comment for EBSR-4) \\
\hline EBSR-4D & 445459069153003 & $\begin{array}{l}\text { East Branch } \\
\text { Sebasticook River }\end{array}$ & 208.34 & (See comment for EBSR-4) \\
\hline $\begin{array}{c}\text { EBSR-5 } \\
\text { (5a) }\end{array}$ & 445452069152701 & $\begin{array}{l}\text { East Branch } \\
\text { Sebasticook River }\end{array}$ & $\begin{array}{l}208.20 \\
\text { (a)202.64 }\end{array}$ & Near water treatment plant \\
\hline
\end{tabular}

\section{Surface-water sites}

The seven surface-water-level measuring points include Corundel Lake, Mill Pond and the East Branch Sebasticook River (fig. 1). They are numbered sequentially beginning with Corundel Lake (CL-1), Mill Pond (MP-1), and proceeding in a downstream direction from East Branch Sebasticook River EBSR-1 to EBSR-5 at the Corinna waste-water treatment plant. The exact location of EBSR-4 changed several times during the course of the study period because of vandalism and beaver activity.

\section{Water-level data}

All hydrologic data collected for this study were obtained between March 19 and June 11, 1999. Ground-water and surface-water level data were collected approximately 10 times during the study period (table 3 ).
Water levels in the twenty wells in the ground-water network were measured using an electric ground-water measuring tape (graduated in tenths of a foot and estimated to hundredths of a foot). Ten of these wells were also instrumented with Telog down-hole data loggers (recording every 15 -minutes, and averaged for each day) to provide continuous records of water-level fluctuations. Daily mean water levels in these wells from March 19, 1999 through June 11, 1999 are provided in Appendix 1.

Water-surface elevations were measured periodically using a ruler or tape (graduated in tenths of a foot and estimated to hundredths of a foot) to determine the distance from a known reference point to the water surface. 
Table 3. Discrete water levels measured in wells and at surface-water sites at the Eastland Woolen Mill Superfund Site, Corinna, Maine, from March to June, 1999

[--, no data; $<$, value is known to be less than value shown]

\begin{tabular}{|c|c|c|c|c|c|c|c|c|c|c|}
\hline \multirow{2}{*}{$\begin{array}{l}\text { Local well number (wells } \\
\text { only) and EPA number }\end{array}$} & \multicolumn{10}{|c|}{ Water level, in feet above sea level } \\
\hline & $\begin{array}{c}\text { Mar. 19, } \\
1999\end{array}$ & $\begin{array}{c}\text { Mar. 26, } \\
1999\end{array}$ & $\begin{array}{l}\text { Apr. 1, } \\
1999\end{array}$ & $\begin{array}{c}\text { Apr. 13, } \\
1999\end{array}$ & $\begin{array}{l}\text { Apr. 23, } \\
1999\end{array}$ & $\begin{array}{c}\text { May 10, } \\
1999\end{array}$ & $\begin{array}{c}\text { May } 14 \\
1999\end{array}$ & $\begin{array}{c}\text { May 25, } \\
1999\end{array}$ & $\begin{array}{c}\text { Jun. 2, } \\
1999\end{array}$ & $\begin{array}{c}\text { Jun. 11, } \\
1999\end{array}$ \\
\hline \multicolumn{11}{|l|}{ GROUND-WATER WELLS } \\
\hline ME-PEW 600; OW-36 & $a_{216.47}$ & 218.14 & 217.70 & 219.30 & 216.82 & 211.03 & $a_{191.12}$ & 215.72 & 204.22 & 216.00 \\
\hline ME-PEW 602; OW-125 & 221.25 & 221.77 & 221.59 & 221.19 & 220.87 & 220.56 & 222.56 & 220.39 & 223.35 & 219.92 \\
\hline ME-PEW 603; OW-59 & 212.57 & 159.85 & 216.21 & 218.10 & 212.23 & 212.02 & 210.38 & 214.41 & 216.56 & 216.33 \\
\hline ME-PEW 604; OW-62 & 224.57 & 224.82 & 225.22 & 224.78 & 224.25 & 223.34 & 223.73 & 223.28 & 222.79 & 223.40 \\
\hline ME-PEW 608; OW-123 & 216.61 & 216.77 & 216.95 & 216.81 & 216.50 & 216.32 & 216.12 & 216.31 & 216.13 & 215.22 \\
\hline ME-PEW 609; 0W-37-1 & $a_{157.71}$ & 84.76 & 124.48 & 153.06 & 153.75 & 111.66 & 101.60 & 150.66 & 144.08 & 111.76 \\
\hline ME-PEW 610; 0W-37-2 & -- & 57.02 & 100.14 & $<95.22$ & 132.97 & 51.82 & $<97.22$ & 109.72 & 60.92 & 122.62 \\
\hline ME-PEW 611; OW-122 (R2) & 215.75 & 215.80 & 215.90 & 215.88 & -- & 215.69 & 215.65 & 215.64 & 215.58 & 215.40 \\
\hline ME-PEW 612; MW-6A & 216.84 & 216.58 & 217.12 & 216.97 & 216.70 & 216.61 & 216.03 & 215.59 & 216.44 & 215.97 \\
\hline ME-PEW 613; MW-6B & 215.25 & 215.84 & 215.86 & 215.14 & 215.33 & 214.37 & 214.25 & 214.28 & 217.27 & 215.11 \\
\hline ME-PEW 614; OW-75 & 235.98 & 234.39 & 237.49 & 236.65 & 235.78 & 230.12 & 235.05 & 235.41 & 226.35 & 235.75 \\
\hline ME-PEW 620; OW-120 & 216.43 & 216.61 & 216.74 & 216.61 & 216.31 & 216.18 & 216.06 & 216.18 & 216.01 & 215.52 \\
\hline \multicolumn{11}{|l|}{ SURFACE-WATER SITES } \\
\hline CL-1 & 228.78 & 228.38 & 228.41 & 228.67 & 228.44 & 228.19 & 228.06 & -- & 227.44 & 229.11 \\
\hline MP-1 & 225.73 & 226.95 & 226.97 & 225.55 & 225.35 & 224.55 & 224.55 & -- & 224.15 & 227.05 \\
\hline EBSR 1 & -- & 216.84 & 218.46 & 216.96 & 217.53 & 216.61 & 216.61 & 216.96 & 216.55 & 217.33 \\
\hline EBSR 2 & 214.91 & 216.64 & 215.83 & 214.96 & 213.46 & 214.16 & 214.05 & -- & 213.90 & 214.75 \\
\hline EBSR 3 & -- & 215.22 & 215.36 & 213.56 & 212.76 & 212.71 & 212.73 & 212.71 & 212.65 & 213.99 \\
\hline EBSR 4 & 208.86 & 208.90 & 209.11 & 206.94 & -- & -- & -- & -- & -- & -- \\
\hline EBSR 4C & -- & -- & -- & -- & -- & 204.34 & 204.35 & 204.25 & 204.10 & 206.15 \\
\hline EBSR 4D & -- & -- & -- & -- & -- & 208.10 & 208.10 & -- & 208.27 & 209.74 \\
\hline EBSR 5 & -- & 204.16 & 204.20 & 203.13 & 201.95 & -- & -- & -- & -- & 203.03 \\
\hline EBSR 5A & -- & -- & -- & -- & -- & 202.01 & 201.99 & 201.99 & 201.89 & -- \\
\hline
\end{tabular}

a - Data for this well is an average of values collected on the given date.

b - Data for this well collected on April 25, 1999

6 Hydrologic Data for the Eastland Woolen Mill Superfund Site, Penobscot County, Corinna, Maine, March through June 1999 


\section{REFERENCES CITED}

Acheron, Inc., 1994, Proposed ground-water pumping plan for downtown Corinna, Maine: Winthrop, Maine, $25 \mathrm{p}$.

Hansen, B.P., Nichols, W.J., and Dudley, R.W., 2001, Borehole geophysical data from Eastland Woolen Mill Superfund Site, Corinna, Maine, March 1999: U. S. Geological Survey Open-File Report 01-186.

Harding Lawson Associates, 1999, Final remedial investigation workplan, Eastland Woolen Mill Site, Corinna, Maine: Portland, Maine, Final Draft August 1999, variously paginated.

Mack, T. J., and Dudley, R. W., 2001, Simulated ground-water-flow responses to geohydrologic characteristics, Corinna, Maine: U. S. Geological Survey Water-Resources Investigations Report WRIR 01-4079.

Osberg, P.H., Hussey, A.M., and Boone, G.M., eds., 1985, Bedrock geologic map of Maine: Maine Geological Survey, Department of Conservation, Augusta, Maine, 1 sheet, scale 1:500,000. 



\section{APPENDIX 1}

DAILY WATER-LEVEL DATA IN WELLS AT THE EASTLAND WOOLEN MILL
SUPERFUND SITE, CORINNA, MAINE, MARCH 19 THROUGH JUNE 11, 1999 
445515069153901, Local number ME-PEW 602; OW-125

ELEVATION (FEET NGVD)， WATER YEAR OCTOBER 1998 TO SEPTEMBER 1999 DAILY MEAN VALUES

\begin{tabular}{|c|c|c|c|c|c|c|c|c|c|c|c|c|}
\hline DAY & OCT & NOV & DEC & JAN & FEB & MAR & $\mathrm{APR}$ & MAY & JUN & JUL & AUG & SEP \\
\hline 1 & --- & --- & --- & --- & --- & --- & 221.55 & 220.75 & --- & --- & --- & --- \\
\hline 2 & --- & --- & --- & --- & --- & --- & 221.56 & 220.50 & --- & --- & --- & --- \\
\hline 3 & --- & --- & --- & --- & --- & --- & 221.62 & 220.37 & --- & --- & --- & --- \\
\hline 4 & --- & --- & --- & --- & --- & --- & 221.53 & 220.38 & --- & --- & --- & --- \\
\hline 5 & --- & --- & --- & --- & --- & --- & 221.34 & 220.57 & --- & --- & --- & --- \\
\hline 6 & --- & --- & --- & --- & --- & --- & 221.38 & 220.58 & --- & --- & --- & --- \\
\hline 7 & --- & --- & --- & --- & --- & --- & 221.27 & 220.40 & --- & --- & --- & --- \\
\hline 8 & --- & --- & --- & --- & --- & --- & 221.31 & 220.35 & --- & --- & --- & --- \\
\hline 9 & --- & --- & --- & --- & --- & --- & 221.25 & 220.50 & --- & --- & --- & --- \\
\hline 10 & --- & --- & --- & --- & --- & --- & 221.42 & --- & --- & --- & --- & --- \\
\hline 11 & --- & --- & --- & --- & --- & --- & 221.09 & --- & --- & --- & --- & --- \\
\hline 12 & --- & --- & --- & --- & --- & --- & 221.13 & --- & --- & --- & --- & --- \\
\hline 13 & --- & --- & --- & --- & --- & --- & 221.16 & --- & --- & --- & --- & --- \\
\hline 14 & --- & --- & --- & --- & --- & --- & 220.95 & --- & --- & --- & --- & --- \\
\hline 15 & --- & --- & --- & --- & --- & --- & 220.98 & --- & --- & --- & --- & --- \\
\hline 16 & --- & --- & --- & --- & --- & --- & 221.18 & --- & --- & --- & --- & --- \\
\hline 17 & --- & --- & --- & --- & --- & --- & 221.04 & --- & --- & --- & --- & --- \\
\hline 18 & --- & --- & --- & --- & --- & --- & 221.11 & --- & --- & --- & --- & --- \\
\hline 19 & --- & --- & --- & --- & --- & --- & 220.65 & --- & --- & --- & --- & --- \\
\hline 20 & --- & --- & --- & --- & --- & 221.32 & 220.47 & --- & --- & --- & --- & --- \\
\hline 21 & --- & --- & --- & --- & --- & 221.18 & 220.44 & --- & --- & --- & --- & --- \\
\hline 22 & --- & --- & --- & --- & --- & 221.49 & 220.70 & --- & --- & --- & --- & --- \\
\hline 23 & --- & --- & --- & --- & --- & 221.83 & 220.71 & --- & --- & --- & --- & --- \\
\hline 24 & --- & --- & --- & --- & --- & 221.67 & 220.88 & --- & --- & --- & --- & --- \\
\hline 25 & --- & --- & --- & --- & --- & 221.51 & 220.68 & --- & --- & --- & --- & --- \\
\hline 26 & --- & --- & --- & --- & --- & 221.64 & 220.56 & --- & --- & --- & --- & --- \\
\hline 27 & --- & --- & --- & --- & --- & 221.76 & 220.73 & --- & --- & --- & --- & --- \\
\hline 28 & --- & --- & --- & --- & --- & 221.65 & 220.64 & --- & --- & --- & --- & --- \\
\hline 29 & --- & --- & --- & --- & --- & 221.73 & 220.62 & --- & --- & --- & --- & --- \\
\hline 30 & --- & --- & --- & --- & --- & 221.57 & 220.71 & --- & --- & --- & --- & --- \\
\hline 31 & --- & --- & --- & --- & --- & 221.53 & --- & --- & --- & --- & --- & --- \\
\hline MEAN & --- & --- & --- & --- & --- & 221.57 & 221.02 & 220.49 & --- & --- & --- & --- \\
\hline
\end{tabular}

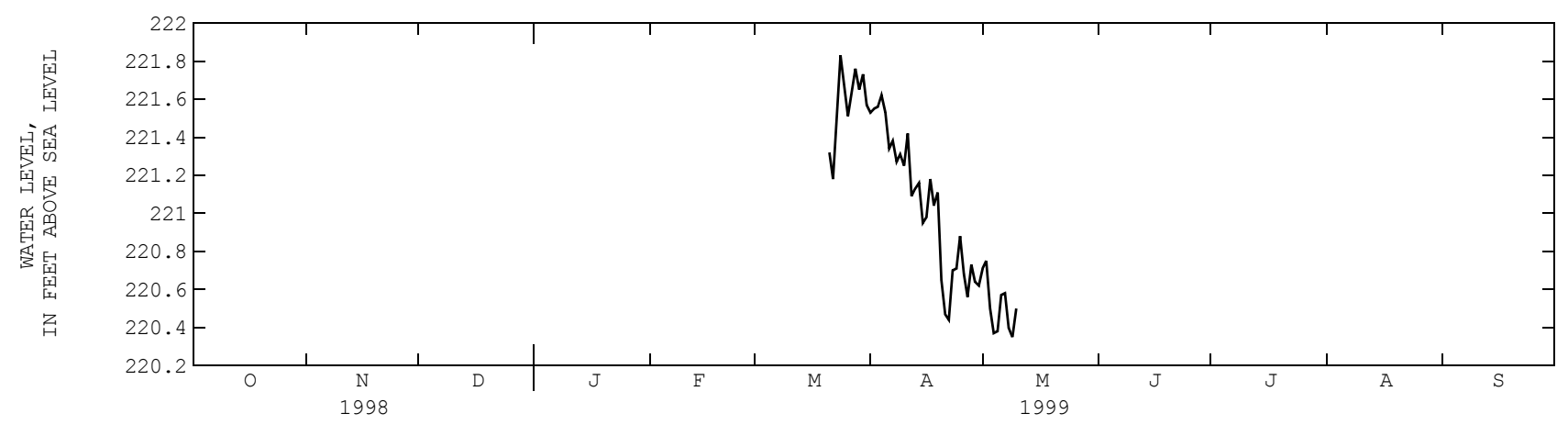


445519069153301, Local number ME-PEW 604; OW-62

ELEVATION (FEET NGVD)，WATER YEAR OCTOBER 1998 TO SEPTEMBER 1999 DAILY MEAN VALUES

\begin{tabular}{|c|c|c|c|c|c|c|c|c|c|c|c|c|}
\hline DAY & OCT & NOV & $\mathrm{DEC}$ & JAN & $\mathrm{FEB}$ & MAR & APR & MAY & JUN & JUL & AUG & SEP \\
\hline 1 & --- & --- & --- & --- & --- & --- & 225.19 & 223.74 & 222.81 & --- & --- & --- \\
\hline 2 & --- & --- & --- & --- & --- & --- & 225.07 & 223.74 & 222.75 & --- & --- & --- \\
\hline 3 & --- & --- & --- & --- & --- & --- & 225.16 & 223.63 & 222.70 & --- & --- & --- \\
\hline 4 & --- & --- & --- & --- & --- & --- & 224.81 & 223.02 & 222.72 & --- & --- & --- \\
\hline 5 & --- & --- & --- & --- & --- & --- & 224.82 & 223.09 & 221.95 & --- & --- & --- \\
\hline 6 & --- & --- & --- & --- & --- & --- & 224.61 & 223.57 & 221.90 & --- & --- & --- \\
\hline 7 & --- & --- & --- & --- & --- & --- & 224.80 & 223.60 & 222.35 & --- & --- & --- \\
\hline 8 & --- & --- & --- & --- & --- & --- & 224.83 & 223.12 & 222.75 & --- & --- & --- \\
\hline 9 & --- & --- & --- & --- & --- & --- & 224.85 & 222.95 & 223.59 & --- & --- & --- \\
\hline 10 & --- & --- & --- & --- & --- & --- & 224.48 & 223.27 & 223.56 & --- & --- & --- \\
\hline 11 & --- & --- & --- & --- & --- & --- & 224.84 & 223.53 & --- & --- & --- & --- \\
\hline 12 & --- & --- & --- & --- & --- & --- & 224.94 & 223.43 & --- & --- & --- & --- \\
\hline 13 & --- & --- & --- & --- & --- & --- & 224.76 & 223.76 & --- & --- & --- & --- \\
\hline 14 & --- & --- & --- & --- & --- & --- & 224.62 & 223.66 & --- & --- & --- & --- \\
\hline 15 & --- & --- & --- & --- & --- & --- & 224.46 & 223.13 & --- & --- & --- & --- \\
\hline 16 & --- & --- & --- & --- & --- & --- & 224.32 & 223.18 & --- & --- & --- & --- \\
\hline 17 & --- & --- & --- & --- & --- & --- & 224.50 & 223.27 & --- & --- & --- & --- \\
\hline 18 & --- & --- & --- & --- & --- & --- & 224.46 & 223.11 & --- & --- & --- & --- \\
\hline 19 & --- & --- & --- & --- & --- & --- & 224.34 & 223.25 & --- & --- & --- & --- \\
\hline 20 & --- & --- & --- & --- & --- & --- & 223.39 & 223.27 & --- & --- & --- & --- \\
\hline 21 & --- & --- & --- & --- & --- & 224.52 & 223.49 & 223.07 & --- & --- & --- & --- \\
\hline 22 & --- & --- & --- & --- & --- & 224.86 & 224.01 & 223.16 & --- & --- & --- & --- \\
\hline 23 & --- & --- & --- & --- & --- & 224.76 & 224.19 & 223.05 & --- & --- & --- & --- \\
\hline 24 & --- & --- & --- & --- & --- & 224.88 & 224.12 & 222.99 & --- & --- & --- & --- \\
\hline 25 & --- & --- & --- & --- & --- & 224.95 & 223.87 & 223.26 & --- & --- & --- & --- \\
\hline 26 & --- & --- & --- & --- & --- & 224.78 & 223.64 & 223.45 & --- & --- & --- & --- \\
\hline 27 & --- & --- & --- & --- & --- & 225.11 & 223.03 & 223.62 & --- & --- & --- & --- \\
\hline 28 & --- & --- & --- & --- & --- & 225.46 & 223.19 & 223.76 & --- & --- & --- & --- \\
\hline 29 & --- & --- & --- & --- & --- & 225.49 & 223.66 & 223.52 & --- & --- & --- & --- \\
\hline 30 & --- & --- & --- & --- & --- & 225.02 & 223.47 & 222.94 & --- & --- & --- & --- \\
\hline 31 & --- & --- & --- & --- & --- & 225.35 & --- & 222.67 & --- & --- & --- & --- \\
\hline MEAN & --- & --- & --- & --- & --- & 225.02 & 224.33 & 223.32 & 222.71 & --- & --- & -- \\
\hline
\end{tabular}

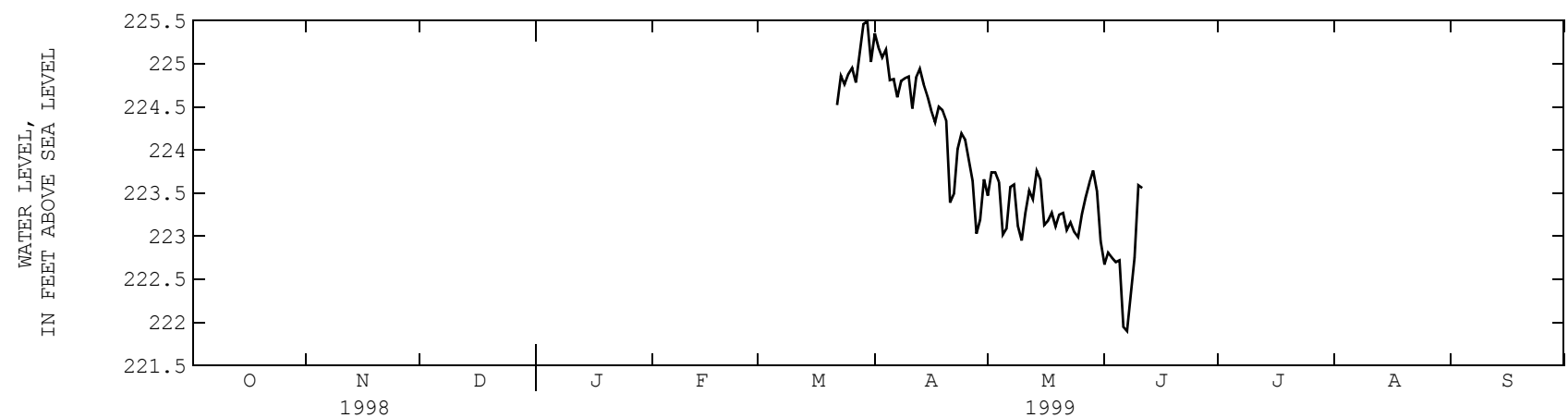




\begin{tabular}{|c|c|c|c|c|c|c|c|c|c|c|c|c|}
\hline \multicolumn{13}{|c|}{ 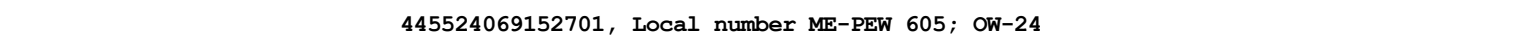 } \\
\hline \multirow[b]{2}{*}{ DAY } & \multirow[b]{2}{*}{ OCT } & \multirow{2}{*}{\multicolumn{2}{|c|}{$\begin{array}{l}\text { ELEVATION } \\
\text { NOV } \quad \text { DEC }\end{array}$}} & \multirow{2}{*}{$\begin{array}{c}\text { (FEET NGVD) , } \\
\text { JAN }\end{array}$} & \multirow{2}{*}{$\begin{array}{l}\text { WATER } \\
\text { DAILY } \\
\text { FEB }\end{array}$} & \multicolumn{2}{|c|}{$\begin{array}{l}\text { YEAR OCTOBER } 1998 \\
\text { MEAN VALUES }\end{array}$} & \multicolumn{3}{|c|}{ TO SEPTEMBER 1999} & \multirow[b]{2}{*}{ AUG } & \multirow[b]{2}{*}{ SEP } \\
\hline & & & & & & MAR & APR & MAY & JUN & JUL & & \\
\hline 1 & --- & --- & --- & --- & --- & --- & 234.45 & 232.97 & 233.47 & --- & --- & --- \\
\hline 2 & --- & --- & --- & --- & --- & --- & 234.41 & 233.63 & 233.26 & --- & --- & --- \\
\hline 3 & --- & --- & --- & --- & --- & --- & 234.48 & 233.76 & 233.10 & --- & --- & --- \\
\hline 4 & --- & --- & --- & --- & --- & --- & 234.36 & 233.78 & 232.96 & --- & --- & --- \\
\hline 5 & --- & --- & --- & --- & --- & --- & 234.34 & 233.80 & 232.74 & --- & --- & --- \\
\hline 6 & --- & --- & --- & --- & --- & --- & 234.31 & 233.71 & 232.64 & --- & --- & --- \\
\hline 7 & --- & --- & --- & --- & --- & --- & 234.34 & 233.84 & 232.79 & --- & --- & --- \\
\hline 8 & --- & --- & --- & --- & --- & --- & 234.39 & 233.95 & 232.89 & --- & --- & --- \\
\hline 9 & --- & --- & --- & --- & --- & --- & 234.36 & 234.01 & 233.55 & --- & --- & --- \\
\hline 10 & --- & --- & --- & --- & --- & --- & 234.42 & 233.91 & 233.73 & --- & --- & --- \\
\hline 11 & --- & --- & --- & --- & --- & --- & 234.53 & 233.77 & --- & --- & --- & --- \\
\hline 12 & --- & --- & --- & --- & --- & --- & 234.41 & 233.55 & --- & --- & --- & --- \\
\hline 13 & --- & --- & --- & --- & --- & --- & 234.36 & 233.45 & --- & --- & --- & --- \\
\hline 14 & --- & --- & --- & --- & --- & --- & 234.30 & 233.27 & --- & --- & --- & --- \\
\hline 15 & --- & --- & --- & --- & --- & --- & 234.24 & 233.34 & --- & --- & --- & --- \\
\hline 16 & --- & --- & --- & --- & --- & --- & 234.29 & 233.23 & --- & --- & --- & --- \\
\hline 17 & --- & --- & --- & --- & --- & --- & 234.40 & 233.15 & --- & --- & --- & --- \\
\hline 18 & --- & --- & --- & --- & --- & --- & 234.38 & 233.21 & --- & --- & --- & --- \\
\hline 19 & --- & --- & --- & --- & --- & --- & 234.33 & 233.23 & --- & --- & --- & --- \\
\hline 20 & --- & --- & --- & --- & --- & 234.39 & 234.27 & 233.40 & --- & --- & --- & --- \\
\hline 21 & --- & --- & --- & --- & --- & 234.32 & 234.25 & 233.44 & --- & --- & --- & --- \\
\hline 22 & --- & --- & --- & --- & --- & 234.27 & 234.20 & 233.49 & --- & --- & --- & --- \\
\hline 23 & --- & --- & --- & --- & --- & 234.30 & 234.25 & 233.24 & --- & --- & --- & --- \\
\hline 24 & --- & --- & --- & --- & --- & 234.35 & 234.26 & 233.24 & --- & --- & --- & --- \\
\hline 25 & --- & --- & --- & --- & --- & 234.34 & 234.21 & 233.74 & --- & --- & --- & --- \\
\hline 26 & --- & --- & --- & --- & --- & 234.27 & 234.20 & 233.80 & --- & --- & --- & --- \\
\hline 27 & --- & --- & --- & --- & --- & 234.37 & 234.06 & 233.76 & --- & --- & --- & --- \\
\hline 28 & --- & --- & --- & --- & --- & 234.42 & 233.90 & 233.74 & --- & --- & --- & --- \\
\hline 29 & --- & --- & --- & --- & --- & 234.42 & 233.77 & 233.87 & --- & --- & --- & --- \\
\hline 30 & --- & --- & --- & --- & --- & 234.33 & 232.38 & 233.91 & --- & --- & --- & --- \\
\hline 31 & --- & --- & --- & --- & --- & 234.42 & --- & 233.69 & --- & --- & --- & --- \\
\hline MEAN & --- & --- & --- & --- & --- & 234.35 & 234.23 & 233.58 & 233.11 & --- & --- & --- \\
\hline
\end{tabular}

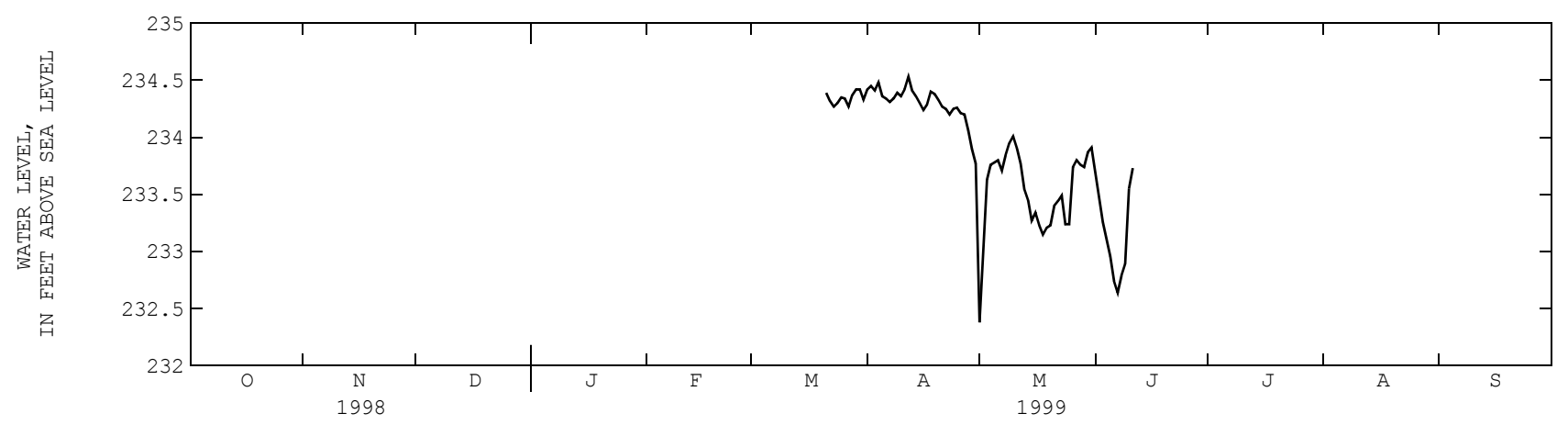

12 Hydrologic Data for the Eastland Woolen Mill Superfund Site, Penobscot County, Corinna, Maine, March through June 1999 
445515069153802, Local number ME-PEW 608; OW-123

ELEVATION (FEET NGVD), WATER YEAR OCTOBER 1998 TO SEPTEMBER 1999 DAILY MEAN VALUES

\begin{tabular}{|c|c|c|c|c|c|c|c|c|c|c|c|c|}
\hline & & & & & & & & & & & & \\
\hline DAY & $\mathrm{OCT}$ & NOV & DEC & JAN & FEB & MAR & $\mathrm{APR}$ & MAY & JUN & JUL & AUG & SEP \\
\hline 1 & --- & --- & --- & --- & --- & --- & 216.95 & 216.54 & 216.09 & --- & --- & --- \\
\hline 2 & --- & --- & --- & --- & --- & --- & 216.98 & 216.44 & 216.09 & --- & --- & --- \\
\hline 3 & --- & --- & --- & --- & --- & --- & 217.02 & 216.39 & 216.14 & --- & --- & --- \\
\hline 4 & --- & --- & --- & --- & --- & --- & 216.95 & 216.38 & 216.11 & --- & --- & --- \\
\hline 5 & --- & --- & --- & --- & --- & --- & 216.80 & 216.35 & 216.05 & --- & --- & --- \\
\hline 6 & --- & --- & --- & --- & --- & --- & 216.82 & 216.36 & 216.05 & --- & --- & --- \\
\hline 7 & --- & --- & --- & --- & --- & --- & 216.82 & 216.33 & 215.94 & --- & --- & --- \\
\hline 8 & --- & --- & --- & --- & --- & --- & 216.82 & 216.30 & 216.06 & --- & --- & --- \\
\hline 9 & --- & --- & --- & --- & --- & --- & 216.78 & 216.33 & 216.13 & --- & --- & --- \\
\hline 10 & --- & --- & --- & --- & --- & --- & 216.73 & 216.27 & 215.45 & --- & --- & --- \\
\hline 11 & --- & --- & --- & --- & --- & --- & 216.69 & 216.17 & --- & --- & --- & --- \\
\hline 12 & --- & --- & --- & --- & --- & --- & 216.75 & 216.21 & --- & --- & --- & --- \\
\hline 13 & --- & --- & --- & --- & --- & --- & 216.79 & 216.18 & --- & --- & --- & --- \\
\hline 14 & --- & --- & --- & --- & --- & --- & 216.75 & 216.11 & --- & --- & --- & --- \\
\hline 15 & --- & --- & --- & --- & --- & --- & 216.71 & 216.14 & --- & --- & --- & --- \\
\hline 16 & --- & --- & --- & --- & --- & --- & 216.69 & 216.17 & --- & --- & --- & --- \\
\hline 17 & --- & --- & --- & --- & --- & --- & 216.72 & 216.19 & --- & --- & --- & --- \\
\hline 18 & --- & --- & --- & --- & --- & --- & 216.69 & 216.20 & --- & --- & --- & --- \\
\hline 19 & --- & --- & --- & --- & --- & --- & 216.52 & 216.20 & --- & --- & --- & --- \\
\hline 20 & --- & --- & --- & --- & --- & --- & 216.42 & 216.21 & --- & --- & --- & --- \\
\hline 21 & --- & --- & --- & --- & --- & 216.57 & 216.37 & 216.17 & --- & --- & --- & --- \\
\hline 22 & --- & --- & --- & --- & --- & 216.77 & 216.41 & 216.13 & --- & --- & --- & --- \\
\hline 23 & --- & --- & --- & --- & --- & 216.86 & 216.48 & 216.16 & --- & --- & --- & --- \\
\hline 24 & --- & --- & --- & --- & --- & 216.82 & 216.52 & 216.15 & --- & --- & --- & --- \\
\hline 25 & --- & --- & --- & --- & --- & 216.83 & 216.50 & 216.27 & --- & --- & --- & --- \\
\hline 26 & --- & --- & --- & --- & --- & 216.76 & 216.50 & 216.28 & --- & --- & --- & --- \\
\hline 27 & --- & --- & --- & --- & --- & 216.86 & 216.46 & 216.24 & --- & --- & --- & --- \\
\hline 28 & --- & --- & --- & --- & --- & 216.87 & 216.41 & 216.23 & --- & --- & --- & --- \\
\hline 29 & --- & --- & --- & --- & --- & 216.96 & 216.45 & 216.20 & --- & --- & --- & --- \\
\hline 30 & --- & --- & --- & --- & --- & 216.93 & 216.50 & 216.16 & --- & --- & --- & --- \\
\hline 31 & --- & --- & --- & --- & --- & 216.91 & --- & 216.14 & --- & --- & --- & \\
\hline MEAN & --- & --- & --- & --- & --- & 216.83 & 216.67 & 216.25 & 216.01 & --- & --- & \\
\hline
\end{tabular}

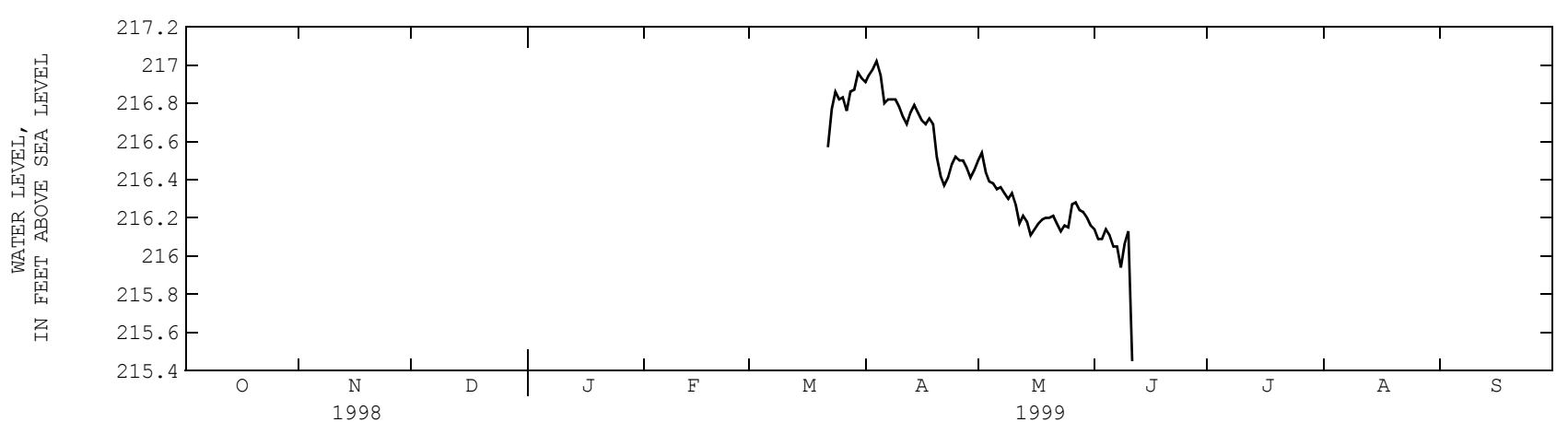


445515069153703, Local number ME-PEW 611; OW-122

ELEVATION (FEET NGVD)， WATER YEAR OCTOBER 1998 TO SEPTEMBER 1999 DAILY MEAN VALUES

\begin{tabular}{|c|c|c|c|c|c|c|c|c|c|c|c|c|}
\hline DAY & OCT & NOV & $\mathrm{DEC}$ & JAN & $\mathrm{FEB}$ & MAR & APR & MAY & JUN & JUL & AUG & SEP \\
\hline 1 & --- & --- & --- & --- & --- & --- & 215.87 & 215.80 & 215.66 & --- & --- & -- \\
\hline 2 & --- & --- & --- & --- & --- & --- & 215.86 & 215.74 & 215.59 & --- & --- & --- \\
\hline 3 & --- & --- & --- & --- & --- & --- & 215.86 & 215.72 & 215.60 & --- & --- & --- \\
\hline 4 & --- & --- & --- & --- & --- & --- & 215.84 & 215.73 & 215.58 & --- & --- & --- \\
\hline 5 & --- & --- & --- & --- & --- & --- & 215.79 & 215.72 & 215.56 & --- & --- & --- \\
\hline 6 & --- & --- & --- & --- & --- & --- & 215.81 & 215.73 & 215.54 & --- & --- & --- \\
\hline 7 & --- & --- & --- & --- & --- & --- & 215.81 & 215.71 & 215.51 & --- & --- & -- \\
\hline 8 & --- & --- & --- & --- & --- & --- & 215.81 & 215.68 & 215.57 & --- & -- & --- \\
\hline 9 & --- & --- & --- & --- & --- & --- & 215.81 & 215.69 & 215.63 & --- & --- & --- \\
\hline 10 & --- & --- & --- & --- & --- & --- & 215.80 & 215.68 & 215.47 & --- & --- & --- \\
\hline 11 & --- & --- & --- & --- & --- & --- & 215.80 & 215.67 & --- & --- & --- & --- \\
\hline 12 & --- & --- & --- & --- & --- & --- & 215.83 & 215.69 & --- & --- & --- & --- \\
\hline 13 & --- & --- & --- & --- & --- & --- & 215.87 & 215.67 & --- & --- & --- & --- \\
\hline 14 & --- & --- & --- & --- & --- & --- & 215.87 & 215.66 & --- & --- & --- & --- \\
\hline 15 & --- & --- & --- & --- & --- & --- & 215.85 & 215.65 & --- & --- & --- & --- \\
\hline 16 & --- & --- & --- & --- & --- & --- & 215.85 & 215.65 & --- & --- & --- & --- \\
\hline 17 & --- & --- & --- & --- & --- & --- & 215.86 & 215.65 & --- & --- & --- & --- \\
\hline 18 & --- & --- & --- & --- & --- & --- & 215.85 & 215.64 & --- & --- & --- & --- \\
\hline 19 & --- & --- & --- & -- & --- & --- & 215.78 & 215.63 & --- & --- & --- & --- \\
\hline 20 & --- & --- & --- & --- & --- & --- & 215.74 & 215.62 & --- & --- & --- & --- \\
\hline 21 & --- & --- & -- & --- & --- & 215.77 & 215.71 & 215.60 & --- & --- & --- & --- \\
\hline 22 & --- & --- & --- & --- & --- & 215.84 & 215.72 & 215.59 & --- & --- & --- & --- \\
\hline 23 & --- & --- & --- & --- & --- & 215.86 & 215.74 & 215.60 & --- & --- & --- & --- \\
\hline 24 & --- & --- & --- & --- & --- & 215.83 & 215.76 & 215.57 & --- & --- & --- & --- \\
\hline 25 & --- & --- & --- & --- & --- & 215.81 & 215.75 & 215.62 & --- & --- & --- & - \\
\hline 26 & --- & --- & --- & --- & --- & 215.79 & 215.74 & 215.54 & --- & --- & --- & --- \\
\hline 27 & --- & --- & --- & --- & --- & 215.84 & 215.72 & 215.74 & --- & --- & --- & --- \\
\hline 28 & --- & --- & --- & --- & --- & 215.87 & 215.72 & 216.00 & --- & --- & --- & --- \\
\hline 29 & --- & --- & --- & --- & --- & 215.91 & 215.75 & 215.89 & --- & --- & --- & --- \\
\hline 30 & --- & --- & --- & --- & --- & 215.89 & 215.77 & 215.81 & --- & --- & -- & -1 \\
\hline 31 & --- & --- & --- & --- & --- & 215.86 & --- & 215.77 & --- & --- & --- & - \\
\hline MEAN & --- & --- & --- & -- & --- & 215.84 & 215.80 & 215.69 & 215.57 & --- & --- & - \\
\hline
\end{tabular}

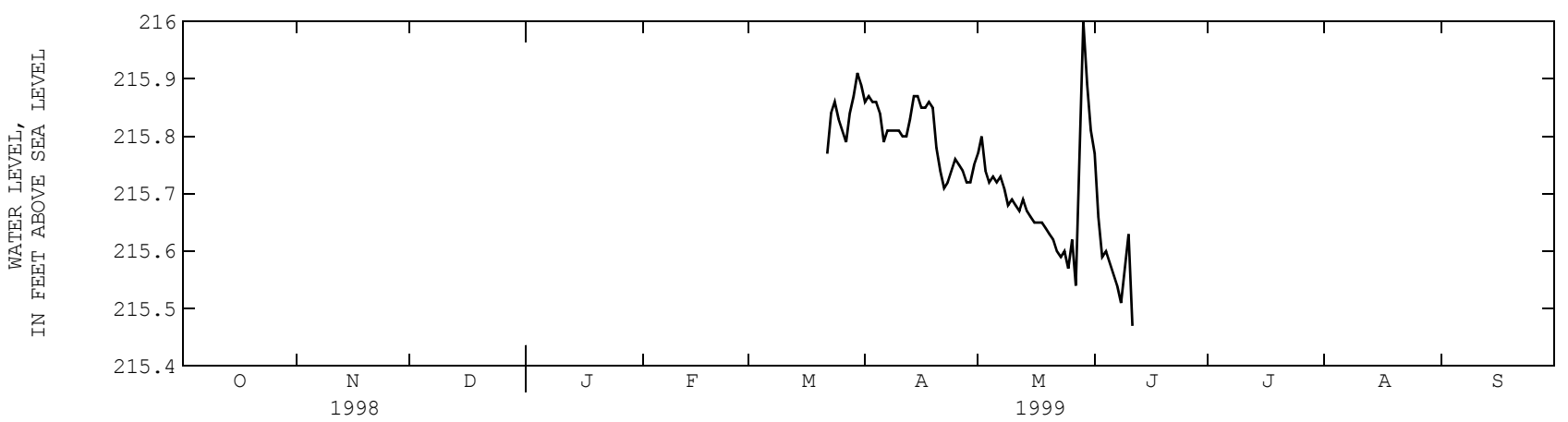

14 Hydrologic Data for the Eastland Woolen Mill Superfund Site, Penobscot County, Corinna, Maine, March through June 1999 
445515069153701, Local number ME-PEW 612; MW-6A

ELEVATION (FEET NGVD), WATER YEAR OCTOBER 1998 TO SEPTEMBER 1999 DAILY MEAN VALUES

\begin{tabular}{|c|c|c|c|c|c|c|c|c|c|c|c|c|}
\hline DAY & OCT & NOV & $\mathrm{DEC}$ & JAN & $\mathrm{FEB}$ & MAR & APR & MAY & JUN & JUL & AUG & SEP \\
\hline 1 & --- & --- & --- & --- & --- & --- & 217.08 & 216.79 & 216.38 & --- & --- & --- \\
\hline 2 & --- & --- & --- & --- & --- & --- & 217.03 & 216.72 & 216.41 & --- & --- & --- \\
\hline 3 & --- & --- & --- & --- & --- & --- & 217.03 & 216.69 & 216.44 & --- & --- & --- \\
\hline 4 & --- & --- & --- & --- & --- & --- & 217.01 & 216.70 & 216.42 & --- & --- & --- \\
\hline 5 & --- & --- & --- & --- & --- & --- & 216.91 & 216.68 & 216.40 & --- & --- & --- \\
\hline 6 & --- & --- & --- & --- & --- & --- & 216.93 & 216.69 & 216.40 & --- & --- & --- \\
\hline 7 & --- & --- & --- & --- & --- & --- & 216.93 & 216.67 & 216.32 & --- & --- & --- \\
\hline 8 & --- & --- & --- & --- & --- & --- & 216.93 & 216.66 & 216.42 & --- & --- & --- \\
\hline 9 & --- & --- & --- & --- & --- & --- & 216.90 & 216.69 & 216.51 & --- & --- & --- \\
\hline 10 & --- & --- & --- & --- & --- & --- & 216.89 & 216.60 & 216.12 & --- & --- & --- \\
\hline 11 & --- & --- & --- & --- & --- & --- & 216.88 & 216.42 & --- & --- & --- & --- \\
\hline 12 & --- & --- & --- & --- & --- & -- & 216.93 & 216.33 & --- & --- & --- & --- \\
\hline 13 & --- & --- & --- & --- & --- & --- & 216.97 & 216.20 & --- & --- & --- & --- \\
\hline 14 & --- & --- & --- & --- & --- & --- & 216.92 & 216.06 & --- & --- & --- & --- \\
\hline 15 & --- & --- & --- & --- & --- & --- & 216.89 & 216.06 & --- & --- & --- & --- \\
\hline 16 & --- & --- & --- & --- & --- & --- & 216.88 & 216.08 & --- & --- & --- & --- \\
\hline 17 & --- & --- & --- & --- & --- & --- & 216.89 & 216.09 & --- & --- & --- & --- \\
\hline 18 & --- & --- & --- & --- & --- & --- & 216.86 & 216.10 & --- & --- & --- & --- \\
\hline 19 & --- & --- & --- & --- & --- & --- & 216.72 & 216.10 & --- & --- & --- & --- \\
\hline 20 & --- & --- & --- & --- & --- & 216.90 & 216.65 & 216.10 & --- & --- & --- & --- \\
\hline 21 & --- & --- & --- & --- & --- & 216.84 & 216.62 & 216.08 & --- & --- & --- & --- \\
\hline 22 & --- & --- & --- & --- & --- & 216.91 & 216.64 & 216.06 & --- & --- & --- & --- \\
\hline 23 & --- & --- & --- & --- & --- & 216.93 & 216.69 & 216.07 & --- & --- & --- & --- \\
\hline 24 & --- & --- & --- & --- & --- & 216.82 & 216.73 & 216.07 & --- & --- & --- & --- \\
\hline 25 & --- & --- & --- & --- & --- & 216.72 & 216.72 & 216.16 & --- & --- & --- & --- \\
\hline 26 & --- & --- & --- & --- & --- & 216.59 & 216.72 & 216.20 & --- & --- & --- & --- \\
\hline 27 & --- & --- & --- & --- & --- & 216.70 & 216.71 & 216.22 & --- & --- & --- & --- \\
\hline 28 & --- & --- & --- & --- & --- & 216.77 & 216.69 & 216.26 & --- & --- & --- & --- \\
\hline 29 & --- & --- & --- & --- & --- & 216.91 & 216.72 & 216.29 & --- & --- & --- & --- \\
\hline 30 & --- & --- & --- & --- & --- & 216.97 & 216.76 & 216.32 & --- & --- & --- & --- \\
\hline 31 & --- & --- & --- & --- & --- & 217.02 & --- & 216.37 & --- & --- & --- & --- \\
\hline MEAN & --- & --- & --- & --- & --- & 216.84 & 216.84 & 216.34 & 216.38 & --- & --- & --- \\
\hline WTR Y & & 216. & & & & & & & & & & \\
\hline
\end{tabular}

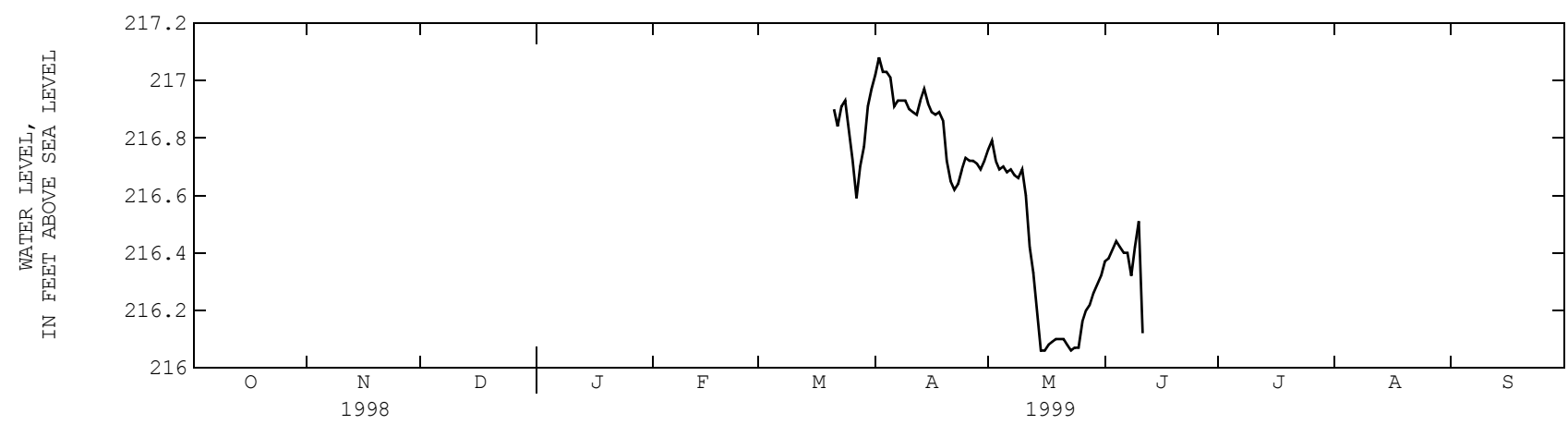


445515069153702, Local number ME-PEW 613; MW-6B

ELEVATION (FEET NGVD)， WATER YEAR OCTOBER 1998 TO SEPTEMBER 1999 DAILY MEAN VALUES

\begin{tabular}{|c|c|c|c|c|c|c|c|c|c|c|c|c|}
\hline DAY & $\mathrm{OCT}$ & NOV & $\mathrm{DEC}$ & JAN & $\mathrm{FEB}$ & MAR & APR & MAY & JUN & JUL & AUG & SEP \\
\hline 1 & --- & --- & --- & --- & --- & --- & 215.83 & 214.74 & 216.93 & --- & --- & --- \\
\hline 2 & --- & --- & --- & --- & --- & --- & 215.68 & 214.68 & 217.13 & --- & --- & --- \\
\hline 3 & --- & --- & --- & --- & --- & --- & 215.40 & 214.63 & 216.80 & --- & --- & --- \\
\hline 4 & --- & --- & --- & --- & --- & --- & 215.35 & 214.56 & 216.48 & --- & --- & --- \\
\hline 5 & --- & --- & --- & --- & --- & --- & 215.33 & 214.54 & 216.14 & --- & --- & --- \\
\hline 6 & --- & --- & --- & --- & --- & --- & 215.31 & 214.53 & 215.80 & --- & --- & --- \\
\hline 7 & --- & --- & --- & --- & -- & --- & 215.27 & 214.46 & 215.52 & --- & --- & --- \\
\hline 8 & --- & --- & --- & --- & --- & --- & 215.16 & 214.40 & 215.90 & --- & --- & --- \\
\hline 9 & --- & --- & --- & --- & --- & --- & 215.16 & 214.42 & 216.40 & --- & --- & --- \\
\hline 10 & --- & --- & --- & --- & --- & --- & 215.16 & 214.36 & 215.49 & --- & --- & --- \\
\hline 11 & --- & --- & --- & --- & --- & --- & 215.17 & 214.30 & --- & --- & --- & --- \\
\hline 12 & --- & --- & --- & --- & --- & --- & 215.14 & 214.25 & --- & --- & --- & --- \\
\hline 13 & --- & --- & --- & --- & -- & --- & 215.15 & 214.20 & --- & --- & --- & --- \\
\hline 14 & --- & --- & --- & --- & --- & --- & 215.18 & 214.18 & --- & --- & --- & --- \\
\hline 15 & --- & --- & --- & --- & --- & --- & 215.22 & 214.17 & --- & --- & --- & --- \\
\hline 16 & --- & --- & --- & --- & --- & --- & 215.30 & 214.17 & --- & --- & --- & --- \\
\hline 17 & --- & --- & --- & --- & --- & --- & 215.35 & 214.16 & --- & --- & --- & --- \\
\hline 18 & --- & --- & --- & --- & --- & --- & 215.43 & 214.16 & --- & --- & --- & --- \\
\hline 19 & --- & --- & --- & --- & --- & --- & 215.39 & 214.17 & --- & --- & --- & --- \\
\hline 20 & --- & --- & --- & --- & --- & --- & 215.13 & 214.18 & --- & --- & --- & --- \\
\hline 21 & --- & --- & --- & --- & --- & 215.24 & 215.13 & 214.17 & --- & --- & --- & --- \\
\hline 22 & --- & --- & --- & --- & --- & 215.82 & 215.20 & 214.17 & --- & --- & --- & --- \\
\hline 23 & --- & --- & --- & --- & -- & 216.28 & 215.26 & 214.17 & --- & --- & --- & --- \\
\hline 24 & --- & --- & --- & --- & --- & 216.08 & 215.21 & 214.23 & --- & --- & --- & --- \\
\hline 25 & --- & --- & --- & --- & --- & 215.96 & 215.13 & 214.40 & --- & --- & --- & --- \\
\hline 26 & --- & --- & --- & --- & --- & 215.81 & 215.04 & 214.67 & --- & --- & --- & --- \\
\hline 27 & --- & --- & --- & --- & -- & 215.78 & 214.96 & 215.06 & --- & --- & --- & --- \\
\hline 28 & --- & --- & --- & --- & --- & 215.79 & 214.91 & 215.43 & --- & --- & --- & --- \\
\hline 29 & --- & --- & --- & --- & --- & 216.05 & 214.84 & 215.80 & --- & --- & --- & --- \\
\hline 30 & --- & --- & --- & --- & --- & 216.00 & 214.79 & 216.20 & --- & --- & --- & --- \\
\hline 31 & --- & --- & --- & --- & --- & 215.91 & --- & 216.58 & --- & --- & --- & --- \\
\hline MEAN & --- & --- & --- & --- & --- & 215.88 & 215.22 & 214.59 & 216.26 & --- & --- & --- \\
\hline
\end{tabular}

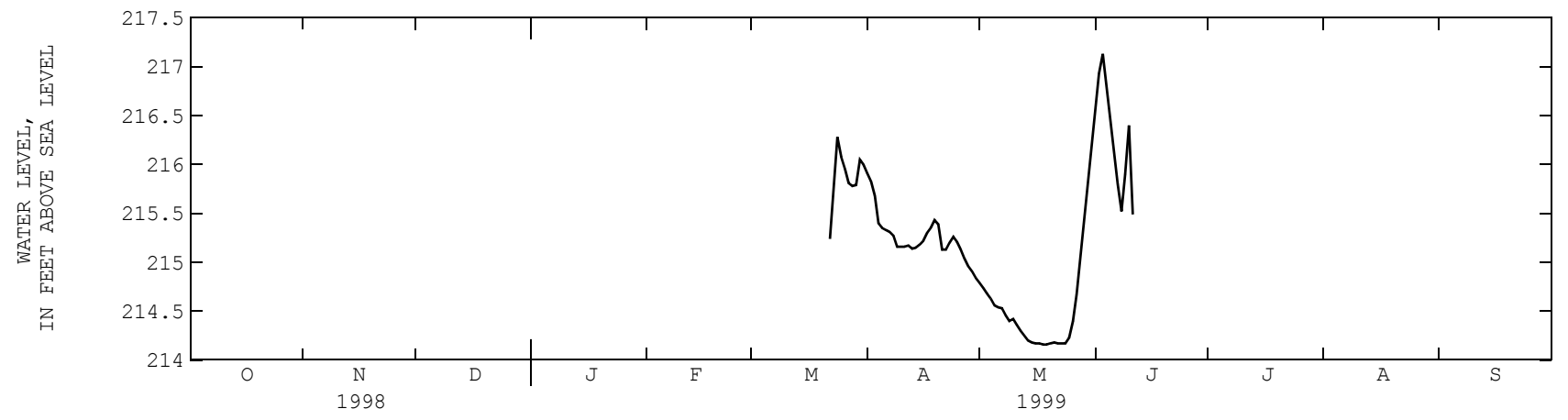


445517069153001, Local number ME-PEW 616; OW-93

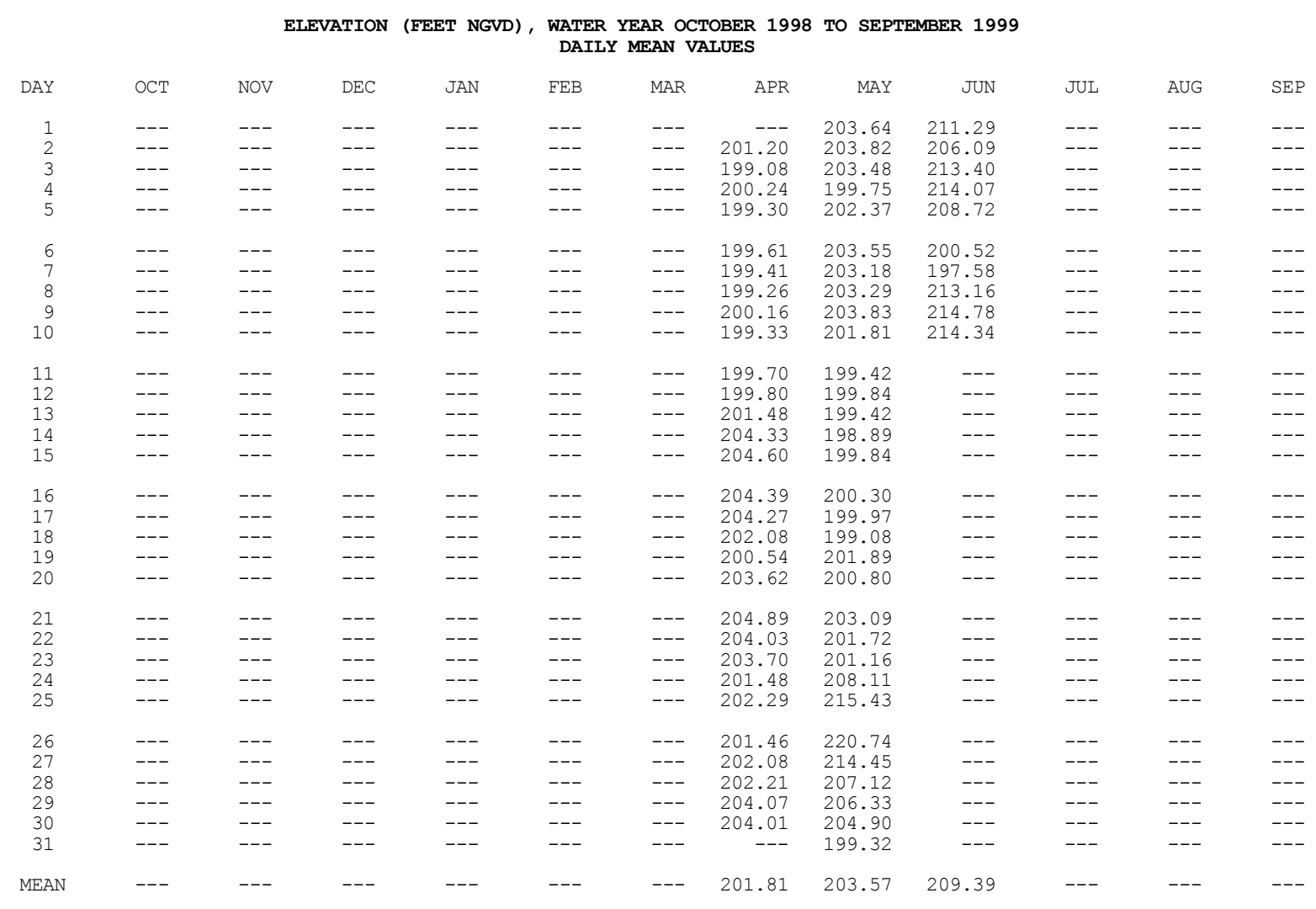

WTR YR 1999 MEAN 203.67
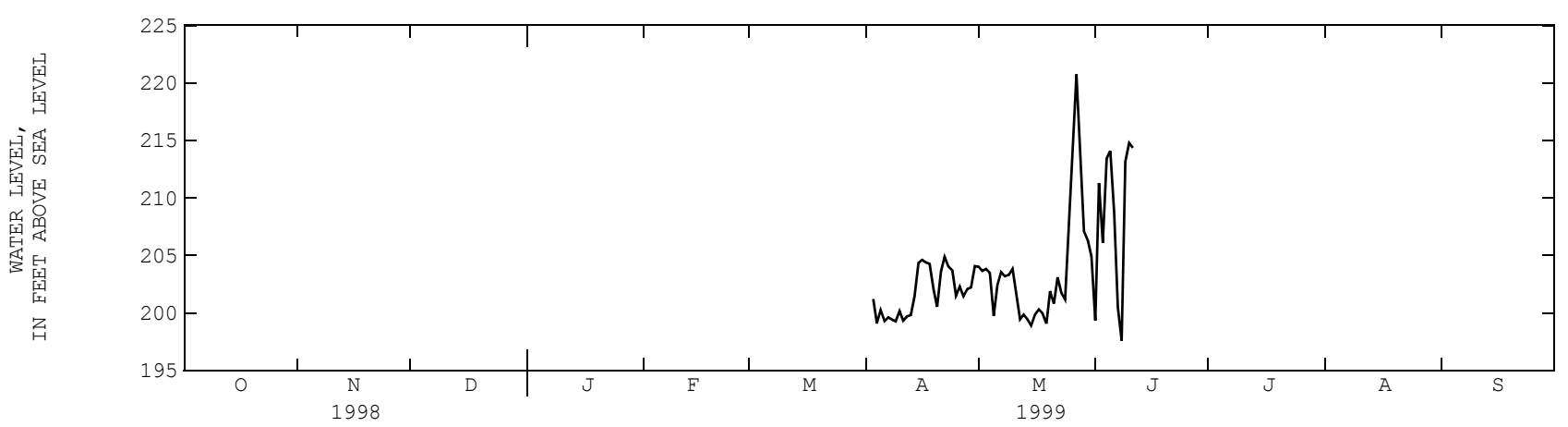
445517069154101, Local number ME-PEW 618; OW-53

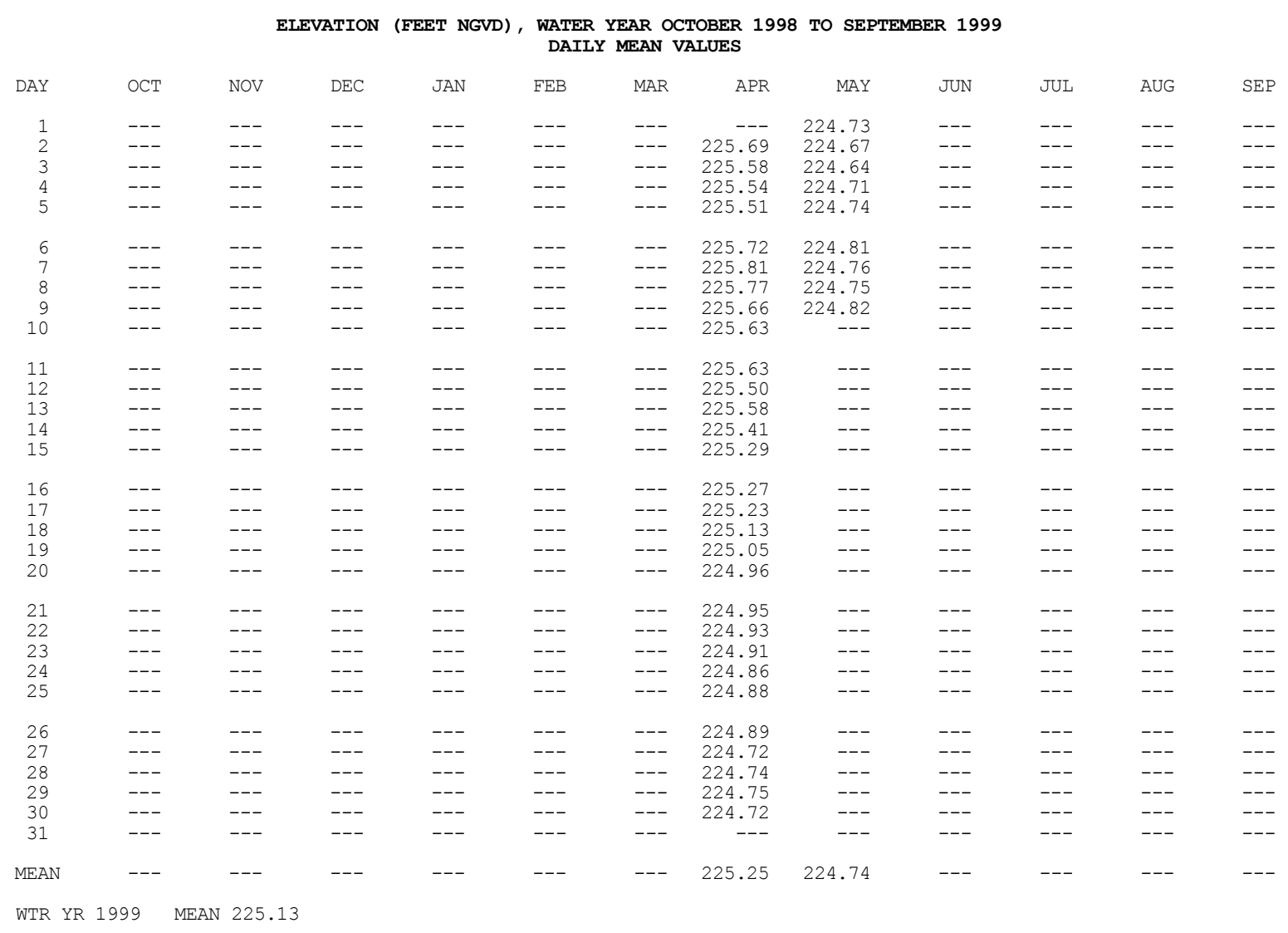

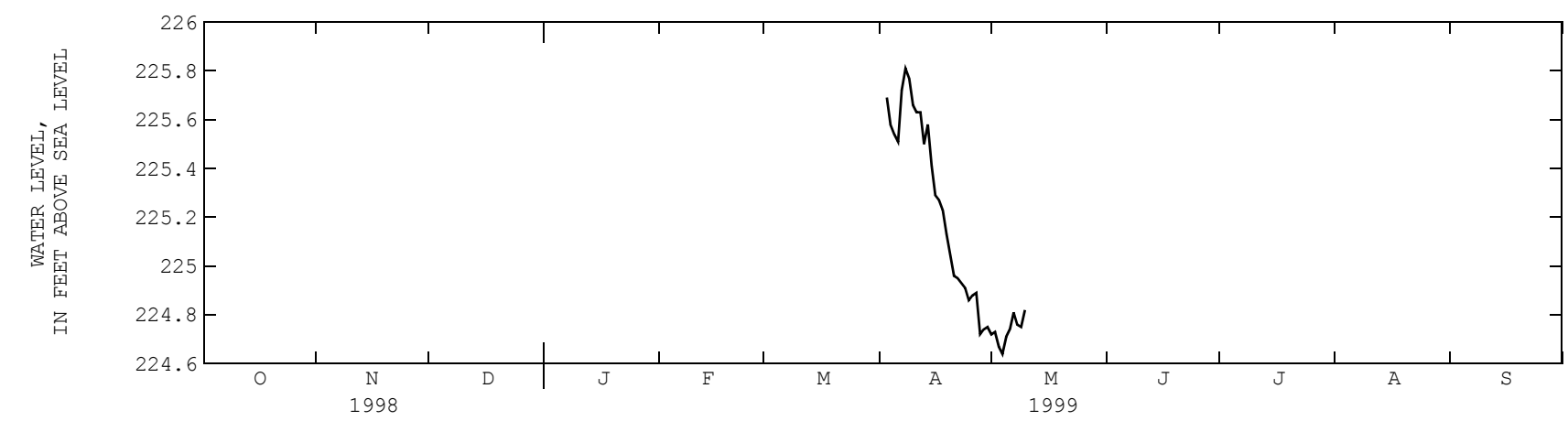

18 Hydrologic Data for the Eastland Woolen Mill Superfund Site, Penobscot County, Corinna, Maine, March through June 1999 
445516069153701, Local number ME-PEW 620; OW-120

ELEVATION (FEET NGVD)， WATER YEAR OCTOBER 1998 TO SEPTEMBER 1999 DAILY MEAN VALUES

\begin{tabular}{|c|c|c|c|c|c|c|c|c|c|c|c|c|}
\hline DAY & $\mathrm{OCT}$ & NOV & $\mathrm{DEC}$ & JAN & FEB & MAR & APR & MAY & JUN & JUL & AUG & SEP \\
\hline 1 & --- & --- & --- & --- & --- & --- & 216.72 & 216.34 & 216.00 & --- & --- & --- \\
\hline 2 & --- & --- & --- & --- & --- & --- & 216.66 & 216.26 & 216.00 & --- & --- & -- \\
\hline 3 & --- & --- & --- & --- & --- & --- & 216.69 & 216.22 & 216.03 & --- & --- & --- \\
\hline 4 & --- & --- & --- & --- & --- & --- & 216.66 & 216.23 & 216.01 & --- & --- & --- \\
\hline 5 & --- & --- & --- & --- & --- & --- & 216.55 & 216.21 & 215.99 & --- & --- & --- \\
\hline 6 & --- & --- & --- & --- & --- & --- & 216.58 & 216.22 & 215.99 & --- & --- & --- \\
\hline 7 & --- & --- & --- & --- & --- & --- & 216.58 & 216.19 & 215.90 & --- & --- & --- \\
\hline 8 & --- & --- & --- & --- & --- & --- & 216.58 & 216.17 & 216.01 & --- & --- & --- \\
\hline 9 & --- & --- & --- & -- & --- & --- & 216.55 & 216.20 & 216.11 & --- & --- & --- \\
\hline 10 & --- & --- & --- & --- & --- & --- & 216.54 & 216.15 & 215.70 & --- & --- & --- \\
\hline 11 & --- & --- & --- & --- & --- & --- & 216.52 & 216.09 & --- & --- & --- & -- \\
\hline 12 & --- & --- & --- & --- & --- & --- & 216.57 & 216.13 & --- & --- & --- & --- \\
\hline 13 & --- & --- & --- & --- & --- & --- & 216.60 & 216.11 & --- & --- & --- & --- \\
\hline 14 & --- & --- & --- & --- & --- & --- & 216.56 & 216.07 & --- & --- & --- & --- \\
\hline 15 & --- & --- & --- & --- & --- & --- & 216.51 & 216.08 & --- & --- & --- & --- \\
\hline 16 & --- & --- & --- & --- & --- & --- & 216.50 & 216.09 & --- & --- & --- & --- \\
\hline 17 & --- & --- & --- & --- & --- & --- & 216.51 & 216.10 & --- & --- & --- & --- \\
\hline 18 & --- & --- & --- & --- & --- & --- & 216.48 & 216.11 & --- & --- & --- & --- \\
\hline 19 & --- & --- & --- & --- & --- & --- & 216.34 & 216.10 & --- & --- & --- & --- \\
\hline 20 & --- & --- & --- & --- & --- & --- & 216.26 & 216.11 & --- & --- & --- & --- \\
\hline 21 & --- & --- & --- & --- & --- & 216.42 & 216.22 & 216.08 & --- & --- & --- & --- \\
\hline 22 & --- & --- & --- & --- & --- & 216.59 & 216.25 & 216.05 & --- & --- & --- & -- \\
\hline 23 & --- & --- & --- & --- & --- & 216.71 & 216.30 & 216.07 & --- & --- & --- & --- \\
\hline 24 & --- & --- & --- & --- & --- & 216.68 & 216.33 & 216.06 & --- & --- & --- & --- \\
\hline 25 & --- & --- & --- & --- & --- & 216.66 & 216.32 & 216.15 & --- & --- & --- & --- \\
\hline 26 & --- & --- & --- & --- & -- & 216.60 & 216.32 & 216.16 & --- & --- & --- & --- \\
\hline 27 & --- & --- & --- & --- & --- & 216.66 & 216.28 & 216.13 & --- & --- & --- & --- \\
\hline 28 & --- & --- & --- & --- & --- & 216.67 & 216.26 & 216.10 & --- & --- & --- & --- \\
\hline 29 & --- & --- & --- & --- & --- & 216.76 & 216.29 & 216.08 & --- & --- & --- & --- \\
\hline 30 & --- & --- & --- & --- & --- & 216.75 & 216.32 & 216.06 & --- & --- & --- & --- \\
\hline 31 & --- & --- & --- & --- & --- & 216.73 & --- & 216.04 & --- & --- & --- & --- \\
\hline MEAN & --- & --- & --- & --- & --- & 216.66 & 216.46 & 216.13 & 215.97 & --- & --- & --- \\
\hline WTR & 199 & N 216 & & & & & & & & & & \\
\hline
\end{tabular}

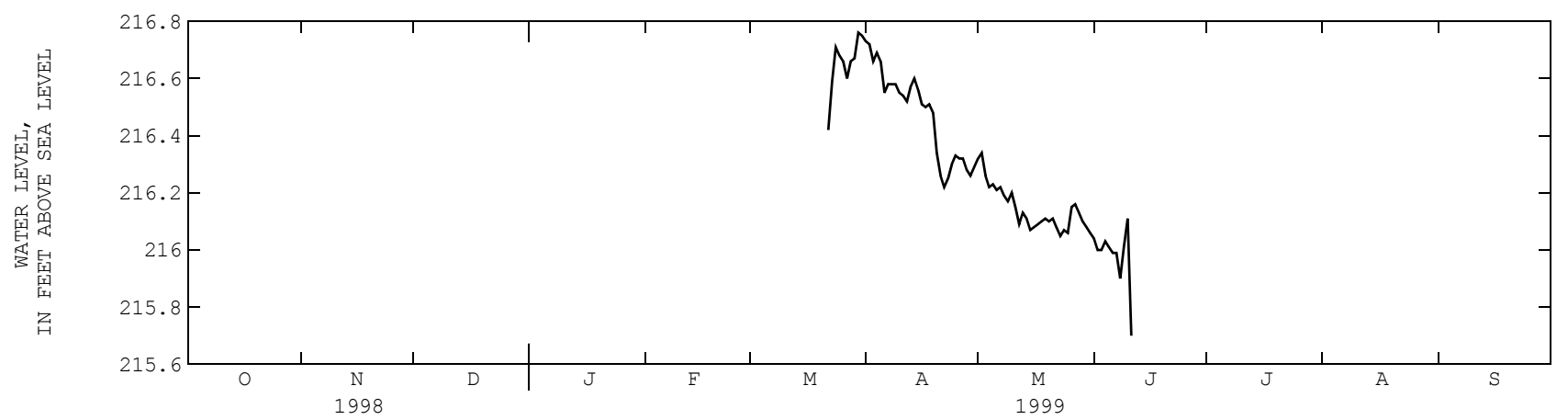



District Chief

Maine District

U.S. Geological Survey

Water Resources Division

26 Ganneston Drive

Augusta, Maine 04330

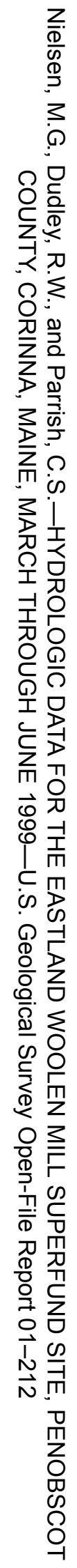

